\title{
Physics and Astrophysics with Ultra-High Energy Cosmic Radiation
}

\author{
Günter Sigl* \\ II. Institut theoretische Physik, Universität Hamburg \\ Luruper Chaussee 149, D-22761 Hamburg, Germany and \\ $\mathrm{APC}^{\dagger}$ (AstroParticules et Cosmologie) \\ 10, rue Alice Domon et Léonie Duquet, 75205 Paris Cedex 13, France \\ E-mail: guenter.sigl@desy.de
}

\begin{abstract}
We give a brief (and highly incomplete) overview of the current experimental and theoretical status of high energy cosmic rays and their secondary $\gamma$-rays and neutrinos. We discuss the role of large scale magnetic fields and the multi-messenger aspects linking these three channels. We recall that the flavor composition of neutrino fluxes from astrophysical sources contains information on both the source conditions and neutrino mixing parameters. Possible effects of new physics beyond the electroweak scale on neutrino detection are recalled. Finally, we discuss the role of Lorentz invariance violation in ultra-high energy cosmic radiation.
\end{abstract}

From Quantum to Emergent Gravity: Theory and Phenomenology

June 11-15 2007

Trieste, Italy

\footnotetext{
*Speaker.

†UMR 7164 (CNRS, Université Paris 7, CEA, Observatoire de Paris)
} 


\section{Introduction}

After almost 90 years of research, the origin of cosmic rays is still an open question, with a degree of uncertainty increasing with energy [1]. Above $\sim 100 \mathrm{MeV}$ the $\mathrm{CR}$ spectrum exhibits little structure and is approximated by broken power laws $\propto E^{-\gamma}$. At the energy $E \simeq 4 \times 10^{15} \mathrm{eV}$ called the "knee", the flux of particles per area, time, solid angle, and energy steepens from a power law index $\gamma \simeq 2.7$ to one of index $\simeq 3.0$. The bulk of the CRs up to at least that energy is believed to originate within the Milky Way Galaxy, typically by shock acceleration in supernova remnants. These objects have been seen in $\gamma$-rays up to $\sim 10 \mathrm{TeV}$ by experiments such as HESS [2] and MAGIC [3], with a spectrum roughly scaling as $E^{-2.2}$. This is consistent with $\gamma$-ray production in interactions with the ambient gas of primary cosmic rays with a similar spectrum at the source. The difference to the cosmic ray spectrum observed to scale as $E^{-2.7}$ below the knee can be explained by diffusion in the galactic magnetic fields.

Above the knee the spectrum continues with a further steepening to $\gamma \simeq 3.3$ at $E \simeq 4 \times 10^{17} \mathrm{eV}$, sometimes called the "second knee". There are experimental indications that the chemical composition changes from light, mostly protons, at the knee to domination by iron and even heavier nuclei at the second knee [4]. This is in fact expected in any scenario where acceleration and propagation is due to magnetic fields whose effects only depend on rigidity, the ratio of charge to rest mass, $Z / A$. This is true as long as energy losses and interaction effects, which in general depend on $Z$ and $A$ separately, are small, as is the case in the Galaxy, in contrast to extra-galactic cosmic ray propagation at ultra-high energy. Above the so called "ankle" or "dip" at $E \simeq 5 \times 10^{18} \mathrm{eV}$, the spectrum flattens again to a power law of index $\gamma \simeq 2.8$. This latter feature is often interpreted as a cross over from a Galactic component, which steepens because cosmic rays are not confined by the galactic magnetic field any more or because Galactic sources do not accelerate beyond the ankle, to a harder component of extragalactic origin. However, the dip at $E \simeq 5 \times 10^{18} \mathrm{eV}$ could also be explained by pair production by extra-galactic protons, if the extra-galactic component already starts to dominate below the ankle, for example, around the second-knee [5] at a few times $10^{17} \mathrm{eV}$. This requires a relatively steep injection spectrum $\propto E^{-2.6-2.7}$. Below a few times $10^{17} \mathrm{eV}$ this extra-galactic component would become unobservable at Earth due to diffusion in extra-galactic magnetic fields (EGMF) [6]. In addition, the effective volume-averaged injection spectrum has to become flatter somewhere below $\sim 10^{18} \mathrm{eV}$ in order to avoid excessive power going into cosmic rays and to avoid overproduction of $\mathrm{GeV}-\mathrm{TeV} \gamma$-rays from $p p$ interactions with the ambient gas.

The low cross-over scenario also requires the dominance of protons around the dip. Theoretically, this can be achieved either because preferentially protons are accelerated or because extended EGMF lead to strong photo-spallation during propagation [7]. Experimentally, above $\simeq 10^{17} \mathrm{eV}$ the chemical composition is basically unknown [8]. Around $10^{18} \mathrm{eV}$ the situation is particularly inconclusive as HiRes [9] and HiRes-MIA [10] data suggest a light (proton dominated) composition, whereas other experiments indicate a heavy composition [4]. In any case, the cosmic ray flux should be extra-galactic at least above the ankle, where a galactic origin would predict an anisotropy toward the galactic plane because galactic magnetic fields can no longer isotropize the cosmic rays. No such anisotropy is seen. There are also experimental indications for a chemical composition becoming again lighter above the ankle, although a significant heavy component is not excluded and the inferred chemical composition above $\sim 10^{18} \mathrm{eV}$ is sensitive to the model of 
air shower interactions and consequently uncertain presently [8]. In addition, should a substantial heavy composition be experimentally observed up to the highest energies, some sources would have to be surprisingly nearby, within a few Mpc, otherwise only low mass spallation products would survive propagation [11]. In the following we will restrict our discussion on extra-galactic ultra-high energy cosmic rays (UHECRs).

No conclusive picture for the nature and distribution of the sources emerges yet naturally from the data [12]: Before 2007, arrival directions appeared approximately isotropic [13], suggesting a large number of weak or distant sources. But there are also indications which point more towards a small number of local and therefore bright sources, especially at the highest energies: First, the AGASA ground array claimed statistically significant multi-plets of events from the same directions within a few degrees [14, 13], although this is controversial [15] and has not been seen so far by other experiments such as the fluorescence experiment HiRes [16]. The spectrum of this clustered component is $\propto E^{-1.8}$ and thus much harder than the total spectrum [14]. Second, nucleons above $\simeq 70 \mathrm{EeV}$ suffer heavy energy losses due to photo-pion production on the cosmic microwave background (CMB) - the Greisen-Zatsepin-Kuzmin (GZK) effect [17] — which limits the distance to possible sources to less than $\simeq 100 \mathrm{Mpc}$ [18]. This predicts a "GZK cutoff", a drop in the spectrum, whose strength depends on the source distribution and may even depend on the part of the sky one is looking at: The "cutoff" could be mitigated in the northern hemisphere where more nearby accelerators related to the local supercluster can be expected. Apart from the SUGAR array which was active from 1968 until 1979 in Australia, all UHECR detectors completed up to the present were situated in the northern hemisphere. Nevertheless the situation is unclear even there: Whereas a "cut-off" is consistent with the few events above $10^{20} \mathrm{eV}$ recorded by the fluorescence detector HiRes [19, 20] and with the second data release of the Pierre Auger observatory [21, 22], there is a tension with the 11 events above $10^{20} \mathrm{eV}$ detected by the AGASA ground array [23]. Still, this could be a combination of statistical and systematic effects [24], especially given the recent downward revision of the energy normalization in AGASA [25]. This question will be solved by the Pierre Auger project which combines the two complementary detection techniques adopted by the aforementioned experiments and whose southern site is near completion in Argentina. Third, about $1 \%$ of the HiRes stereo events around $10^{19} \mathrm{eV}$ seem to correlate with active galaxies of the BL Lac type on a scale of $\sim 0.6^{\circ}$, with a significance of $\sim 10^{-4}[26,27]$. Due to deflection of UHECR in the galactic magnetic field this, however, would have to be neutral primaries that cannot be created in the necessary quantities over the distances involved. Finally, and perhaps most importantly, in 2007 the Pierre Auger collaboration published a paper indicating that the arrival directions of cosmic rays about $6 \times 10^{19} \mathrm{eV}$ correlate with the positions of active galactic nuclei within $75 \mathrm{Mpc}$ distance from Earth [28] which finally establishes an anisotropy following the large scale structure and in particular the supergalactic plane.

\section{Role of large scale magnetic fields}

The hunt for UHECR sources is further complicated by the presence of large scale cosmic magnetic fields which may significantly deflect charged cosmic rays even at the highest energies, in particular if sources correlate with high magnetic field regions such as galaxy clusters and/or if there is a significant component of heavy nuclei at the highest energies. A major issue in UHECR 
propagation studies is, therefore, the strength and distribution of EGMF. Galaxy clusters harbor magnetic fields of $\mu \mathrm{G}$ strength, but it is poorly known how quickly these fields fall off with increasing distance from the cluster center. The current data indicate that $\mu \mathrm{G}$ strength magnetic fields extend to at least $\sim 1 \mathrm{Mpc}$ [29] and possibly to larger distances [30, 31]. Beyond $\simeq 1 \mathrm{Mpc}$ from a cluster core, however, probing the magnetic fields becomes extremely difficult because the Faraday Rotation Measure loses sensitivity in low density regions. Furthermore, the intracluster magnetic field topology is also poorly known, although the situation will likely improve in the future, for example with the advent of powerful radio astronomical instruments such as the square kilometer array.

One possibility in the meantime is to adopt large scale structure simulations (LSS) which include magnetic fields. In Ref. [32], the authors use magnetic fields derived from a cosmological LSS with magnetic fields generated at the shocks that form during LSS formation, whereas in Ref. [33] and Ref. [34] fields of "primordial" origin have been considered. While the different models for initial magnetic seed fields produce different large scale magnetic field distributions and, therefore, lead to different predictions for UHECR deflection, there is still a significant discrepancy between Ref. [32, 33] and Ref. [34], hinting that other technical reasons may play a role here. In the more extended fields from the simulations of Refs. [32, 33] deflection of protons up to $10^{20} \mathrm{eV}$ can be up to tens of degrees, whereas deflections in the simulations of Ref. [34] are typically below a degree. Assuming the EGMF correlates with the infrared luminosity density, Ref. [35] recently found results closer to Refs. [32, 33] than Ref. [34].

We recall that since acceleration is rigidity dependent, at the acceleration sites the highest energy cosmic ray flux is likely dominated by heavy nuclei. If this is indeed the case, it is interesting to point out that even in the EGMF scenario of Ref. [34], deflections could be considerable. One can in fact define a critical energy $E_{c}$ below which UHECR will largely be in the diffusive regime, whereas they will propagate roughly ballistically at much higher energies. For an UHECR of charge $Z$ propagating over a distance scale $d$ in a field of strength $B$ and coherence length $\lambda_{c}$ this energy can be estimated as

$$
E_{\mathrm{c}} \sim 1.2 \times 10^{19}\left(\frac{Z}{26}\right)\left(\frac{d}{\mathrm{Mpc}}\right)^{1 / 2}\left(\frac{B_{\mathrm{rms}}}{\mathrm{nG}}\right)\left(\frac{\lambda_{c}}{1 \mathrm{Mpc}}\right)^{1 / 2} \mathrm{eV}
$$

In contrast to the contribution of our Galaxy to deflection which can be of comparable size but may be corrected for within sufficiently detailed models of the galactic field, the extra-galactic contribution would be stochastic. Statistical methods are therefore likely to be necessary to learn about UHECR source distributions and characteristics as well as EGMF. For example, a suppressed UHECR arrival direction auto-correlation function at degree scales, rather than pointing to a high source density, could be a signature of extended EGMF [32].

Finally, EGMF can considerably increase the path-length of UHECR propagation and thus modify spectra, especially from individual sources, as well as the chemical composition observed at Earth [36]. 


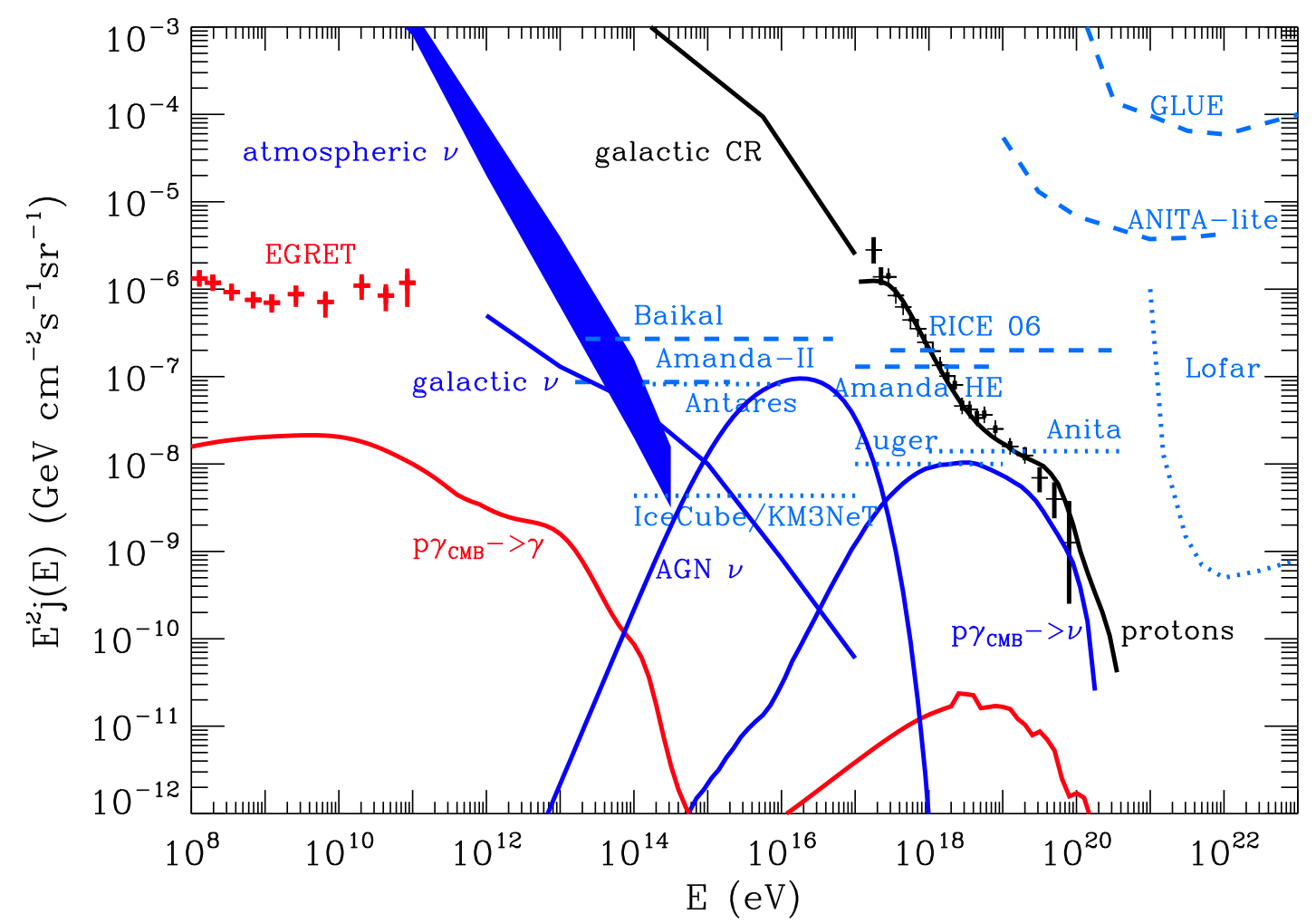

Figure 1: Model fluxes compared to experimental data, limits and sensitivities. Primary cosmic ray fluxes (data and a model, see text) are shown in black, the secondary $\gamma$-ray flux expected from proton interactions with the CMB and infrared background in red and the "guaranteed" neutrino fluxes per neutrino flavor in blue: atmospheric neutrinos, galactic neutrinos resulting from cosmic ray interactions with matter in our Galaxy [46], and "GZK" neutrinos resulting from cosmic ray interaction with the CMB and infrared background. The GZK neutrino fluxes depend on the distribution of the (unknown) primary cosmic ray sources for which we assumed active galactic nuclei (AGNs) above $10^{17} \mathrm{eV}$, using our public CRPropa code [41, 42]. Cosmic ray interactions within these sources can also produce neutrinos for which one example is given (AGN $v$ ) [43]. The flux of atmospheric neutrinos has been measured by underground detectors and AMANDA. The dashed and dotted blue lines are existing upper limits and future sensitivities to diffuse neutrino fluxes from various experiments, respectively [37], assuming the Standard Model neutrinonucleon cross section extrapolated to the relevant energies. The maximum possible neutrino flux would be given by horizontally extrapolating the diffuse $\gamma$-ray background observed by EGRET [47].

\section{Multi-messenger approach: Secondary gamma-rays and neutrinos and their flavor composition}

The physics and astrophysics of UHECRs are also intimately linked with the emerging field of neutrino astronomy [37] as well as with the already well established field of $\gamma$-ray astronomy [38]. Indeed, all scenarios of cosmic ray origin, from the galactic scale [39] to top-down [40] and Z-burst models at the highest energies [43], are severely constrained by neutrino and $\gamma$-ray observations and limits. This has, for example, important consequences for theoretical predictions of diffuse fluxes of extragalactic neutrinos above about a $\mathrm{TeV}$ whose detection is a major goal 
of next-generation neutrino telescopes: If these neutrinos are produced as secondaries of protons accelerated in astrophysical sources and if these protons leave the sources and contribute to the UHECR flux observed, then the energy content in the neutrino flux can not be higher than the one in UHECRs, leading to the so called Waxman-Bahcall bound for transparent sources with soft acceleration spectra $[44,45]$. If one of these assumptions does not apply, such as for acceleration sources with injection spectra harder than $E^{-2}$ and/or opaque to nucleons, or if much fewer nucleons than $\gamma$-rays and neutrinos are produced, such as in top-down scenarios, the Waxman-Bahcall bound does not apply, but the neutrino flux is still constrained by the observed diffuse $\gamma$-ray flux in the $\mathrm{GeV}$ range.

Fig. 1 provides a sketch of "realistic" cosmic ray, $\gamma$-ray, and neutrino flux predictions in comparison with experimental observations, limits, and sensitivities. It shows a theoretical scenario in which extra-galactic cosmic ray sources roughly evolving as quasars inject a spectrum $\propto E^{-2.6}$ of dominantly protons down to $\sim 10^{17} \mathrm{eV}$ where a cross-over to galactic cosmic rays occurs [5]. The "cosmogenic" neutrino flux produced by protons interacting with the low energy photon background considerably depends on these assumptions which can thus be used to test them [48].

Apart from cosmogenic neutrinos produced during propagation of UHECR, neutrinos can also be produced within astrophysical sources such as AGNs (see Fig. 1) or $\gamma$-ray bursts. In the absence of matter effects, a source at cosmological distances injecting neutrino fluxes with a flavor ratio $\propto w_{\beta}, \beta=e, \mu, \tau$, leads to a flavor mixture $\phi_{\alpha} \propto \sum_{\beta, i} w_{\beta}\left|U_{\alpha i}\right|^{2}\left|U_{\beta i}\right|^{2}$ observed at Earth, where $U_{\alpha i}$ is the mixing matrix and $i$ labels mass eigenstates. Therefore, if both pions and muons decay before loosing energy around the source, $w_{e}: w_{\mu}: w_{\tau} \simeq 1: 2: 0$ and thus $\phi_{e}: \phi_{\mu}: \phi_{\tau} \simeq 1: 1: 1$. At high energies the meson and muon energy loss time $t_{\text {loss }}(E)$ becomes shorter than their decay time $E \tau / m$, and the neutrino spectrum will be suppressed by a factor $\simeq m t_{\text {loss }}(E) /(\tau E)$ compared to primary interaction rates. For hadronic cooling, $t_{\text {loss }} \sim$ const, whereas for radiative cooling at the highest energies, $t_{\text {loss }}(E) \propto E^{-1}$, resulting in a steepening of the neutrino spectrum by a factor $E^{-1}$ and $E^{-2}$, respectively [49]. In addition, at a given energy, charged pions decay about hundred times faster than muons. There can thus be an energy range at which pions but not muons decay before loosing energy such that $w_{e}: w_{\mu}: w_{\tau} \simeq 0: 1: 0$ and thus $\phi_{e}: \phi_{\mu}: \phi_{\tau} \simeq 1: 2: 2$. Also, $p p$ interactions produce both pions of both charges and thus give a higher fraction of $\bar{v}_{e}$ compared to $p \gamma$ interactions. The observed flavor ratios can thus depend on energy and carry information on the source conditions [50], but also about the mixing matrix itself [51].

Experimentally, different flavors can be distinguished by the different signatures their charged current interactions cause in the detectors [37]: Muon neutrinos tend to produce long muon tracks whose Cherencov cones can be detected optically. Electron neutrinos produce electromagnetic and hadronic showers from the elecrons and nuclear recoils, respectively, which they give rise to. Finally, tau neutrinos can give rise to "double bang" signatures where the first "bang" is produced by the recoil of the nucleus on which the charged current reaction took place, and the second "bang" is due to the tau lepton decay. This signature is visible at energies for which the tau lepton deay length is comparable to the detector dimensions. Given sufficient statistics, these different signatures should make it possible to distinguish between the scenarios discussed above.

Finally, flavor ratios can probe new physics, such as neutrino decay and quantum decoherence [52]: If all but the lightest mass eigenstate $j$ decay before reaching the observer, the flux of flavor $\alpha$ observed at Earth would be $\propto\left|U_{\alpha j}\right|^{2}$, independent of the flavor ratio at the source. For 
$j=1$ (normal mass hierarchy) this gives $\phi_{e}: \phi_{\mu}: \phi_{\tau} \simeq 6: 1: 1$, whereas for $j=3$ (inverted mass hierarchy) one has $\phi_{e}: \phi_{\mu}: \phi_{\tau} \simeq 0: 1: 1$, which should be easy to distinguish from the normal case. This would allow to probe lifetimes of the order $\tau / m \sim 300(E / P e V)^{-1} \mathrm{~s} / \mathrm{eV}$, which could improve on current limits. Quantum decoherence would predict $\phi_{e}: \phi_{\mu}: \phi_{\tau}=1: 1: 1$, independent of source flavor ratios.

\section{Physics beyond the electroweak scale: New neutrino interactions}

Within the Standard Model the interaction cross section of neutrinos with nucleons falls short by about five orders of magnitude to produce air showers starting high in the atmosphere as observed. Electroweak instantons could change this but this possibility is speculative [53]. The neutrino-nucleon cross section, however, can be enhanced by new physics beyond the electroweak scale in the center of mass (CM) frame, or above about a PeV in the nucleon rest frame. Note that the CM energy reached by an UHECR particle of energy $E$ interacting with an atmospheric nucleon at rest is $\sqrt{s} \simeq 0.4\left(E / 10^{20} \mathrm{eV}\right) \mathrm{PeV}$. While physics beyond the electroweak scale would influence the interactions of all particles, its effects would be burried below the strong interactions for hadron primaries. In contrast, neutrino interactions would be a much more sensitive probe to new physics because their Standard Model interactions are much weaker. Neutrino induced air showers may therefore rather directly probe new physics beyond the electroweak scale.

One possibility consists of a large increase in the number of degrees of freedom above the electroweak scale [54]. A specific instance of this idea appears in theories with additional large compact dimensions and a quantum gravity scale in the $\mathrm{TeV}$ range that has recently received much attention in the literature [55] because it provides an alternative solution to the hierarchy problem in grand unifications of gauge interactions without a need of supersymmetry. In such scenarios microscopic black holes and even higher dimensional branes can be produced by interactions of neutrinos with one of the constituent partons of the nucleon at center of mass energies above the fundamental quantum gravity scale. The resulting total neutrino-nucleon cross sections can be larger than in the Standard Model by up to a factor $\sim 100$ if the fundamental gravity scale is of order $\mathrm{TeV}$ [56].

Whereas the sub-hadronic scale cross sections obtained in some extra dimension scenarios are still too small to be consistent with observed air showers and thus to explain the observed UHECR events [57], they can still have important phenomenological consequences. This is because UHECR data can be used to put constraints on neutrino nucleon cross sections smaller than $\lesssim 10^{-27} \mathrm{~cm}^{2}$. Particles with such cross sections would give rise to horizontal air showers which have not yet been observed. Resulting upper limits on their fluxes assuming the Standard Model cross section are shown in Fig. 1. Comparison with the "cosmogenic" neutrino flux produced by UHECRs interacting with the CMB then results in upper limits on the cross section which are about a factor of few hundred larger than the Standard Model cross section in the energy range between $\simeq 10^{17} \mathrm{eV}$ and $\simeq 10^{19} \mathrm{eV}[58,59,60]$. The projected sensitivity of future experiments also shown in Fig. 1 indicate that these limits could be lowered down to the Standard Model cross section [60]. In case of a detection of penetrating events the degeneracy of the cross section with the unknown neutrino flux could be broken by comparing the rates of horizontal air showers with the ones of 
Earth skimming events [61]. This would allow to "measure" the neutrino-nucleon cross section at energies unreachable by any forseeable terrestrial accelerator !

\section{Violation of Lorentz Invariance}

A number of authors pointed out $[62,63]$ that there may be no GZK effect if Lorentz invariance is violated by a tiny amount that is consistent with all current experiments. At a purely theoretical level, several quantum gravity models including some based on string theories do in fact predict non-trivial modifications of space-time symmetries that also imply VLI at extremely short distances (or equivalently at extremely high energies); see e.g., Ref. [64] and references therein. These theories are, however, not yet in forms definite enough to allow precise quantitative predictions of the exact form of the possible violation of Lorentz invariance (VLI). Current formulations of the effects of a possible VLI on high energy particle interactions relevant in the context of UHECR, therefore, adopt a phenomenological approach in which the form of the possible VLI is parametrized in various ways, usually within effective field theory which characterizes possible terms allowed at energies much lower than the grand unification and Planck scales, without explicit reference to their origin in any theory describing physics at these much higher energies. VLI generally implies the existence of a universal preferred frame which is usually identified with the frame that is comoving with the expansion of the Universe, in which the CMB is isotropic.

A direct way of introducing VLI is through a modification of the standard dispersion relation, $E^{2}-p^{2}=m^{2}$, between energy $E$ and momentum $p=|\vec{p}|$ of particles, $m$ being the invariant mass of the particle. Currently there is no unique way of parameterizing the possible modification of this relation in a Lorentz non-invariant theory. We discuss here a parameterization of the modified dispersion relation which covers most of the qualitative cases discussed in the literature and, for certain parameter values, allows to completely evade the GZK limit in case of nucleons,

$$
E^{2}-p^{2}-m^{2} \simeq-2 d E^{2}-\xi \frac{E^{3}}{M_{\mathrm{Pl}}}-\zeta \frac{E^{4}}{M_{\mathrm{Pl}}^{2}} .
$$

Here, the Planck mass $M_{\mathrm{Pl}}$ characterizes non-renormalizable effects with dimensionless coefficients $\xi$ and $\zeta$, and the dimensionless constant $d$ exemplifies VLI effects due to renormalizable terms in the Lagrangian. The standard Lorentz invariant dispersion relation is recovered in the limit $\xi, \zeta, d \rightarrow 0$.

The constants $d \neq 0$ can break Lorentz invariance spontaneously when certain Lorentz tensors $c_{\mu \nu}$ have couplings to fermions of the form $d_{\mu \nu} \bar{\psi} \gamma^{\mu} \partial^{v} \psi$, and acquire vacuum expectation values of the form $\left\langle d_{\mu \nu}\right\rangle=d \delta_{\mu}^{0} \delta_{v}^{0}$. If rotational invariance and gauge symmetry are preserved, such renormalizable Lorentz invariance breaking terms in the Lagrangian are characterized by a single timelike vector $u^{\mu}$, with $u^{\mu} u_{\mu}=-1$, which defines a preferred reference frame [65]. The dimensionless terms can be interpreted as a change of the maximal particle velocity [63] $v_{\max }=\partial E /\left.\partial p\right|_{E, p \gg m} \simeq$ $1-d$. At a fixed energy $E$ one has the correspondence $d \rightarrow(\xi / 2)\left(E / M_{\mathrm{Pl}}\right)+(\zeta / 2)\left(E / M_{\mathrm{Pl}}\right)^{2}$, as can be seen from Eq. (5.1).

Within effective field theory, effects of first order in $M_{\mathrm{Pl}}^{-1}, \xi \neq 0$, arise from the most general terms of the form

$$
\frac{\kappa}{2 M_{\mathrm{Pl}}} u^{\mu} F_{\mu v}(u \cdot \partial) u_{\lambda} \tilde{F}^{\lambda v}+\frac{1}{2 M_{\mathrm{Pl}}} u^{\mu} \bar{\psi} \gamma_{\mu}\left(\lambda_{1}+\lambda_{2} \gamma_{5}\right)(u \cdot \partial)^{2} \psi
$$


where $\tilde{F}$ denotes the dual of the field strength $F$. For photons and electrons this leads to $\xi_{ \pm}= \pm \kappa$ and $\xi_{ \pm}=\lambda_{1} \pm \lambda_{2}$, respectively, in Eq. (5.1), where \pm refers to helicity which remains conserved in the presence of the terms Eq. (5.2) [66]. These terms also violate CPT and are, therefore, sometimes considered to be unlikely to be present [67]. However, effects of first order in $M_{\mathrm{Pl}}^{-1}, \xi \neq 0$, are possible, for example, in non-critical Liouville string theory due to recoiling D-branes [68], where sub-luminal propagation, i.e. $\xi<0$ seems to be preferred. Finally, in critical string theory, effects second order in $M_{\mathrm{Pl}}^{-1}, \zeta \neq 0$, can be induced due to quantum gravity effects.

Note that in supersymmetric QED, corrections to the dispersion relation of a particle of mass $m$ are of the form $\xi_{n} m^{2}\left(k / M_{\mathrm{Pl}}\right)^{n}$ and are thus negligible in astrophysical contexts [69]. Therefore, significant constraints can only be obtained in the non-supersymmetric case.

Now, consider the GZK photo-pion production process in which a nucleon of energy $E$, momentum $p$ and mass $m_{N}$ collides head-on with a CMB photon of energy $\varepsilon$ producing a pion and a recoiling nucleon. The threshold initial momentum of the nucleon for this process according to standard Lorentz invariant kinematics is

$$
p_{\text {th }, 0}=\left(m_{\pi}^{2}+2 m_{\pi} m_{N}\right) / 4 \varepsilon,
$$

where $m_{\pi}$ and $m_{N}$ are the pion and nucleon masses, respectively. Assuming exact energy-momentum conservation but using the modified dispersion relation Eq. (5.1) for $d=0$ for nucleons, in the ultra-relativistic regime $m \ll p \ll M$, and neglecting sub-leading terms, the new nucleon threshold momentum $p_{\text {th }}$ satisfies [70]

$$
-\beta x^{4}-\alpha x^{3}+x-1=0
$$

where $x=p_{\text {th }} / p_{\text {th }, 0}$, and

$$
\begin{aligned}
& \alpha=\frac{2 \xi p_{\mathrm{th}, 0}^{3}}{\left(m_{\pi}^{2}+2 m_{\pi} m_{N}\right) M_{\mathrm{Pl}}} \frac{m_{\pi} m_{N}}{\left(m_{\pi}+m_{N}\right)^{2}}, \\
& \beta=\frac{3 \zeta p_{\mathrm{th}, 0}^{4}}{2\left(m_{\pi}^{2}+2 m_{\pi} m_{N}\right) M_{\mathrm{Pl}}^{2}} \frac{m_{\pi} m_{N}}{\left(m_{\pi}+m_{N}\right)^{2}} .
\end{aligned}
$$

Here, $\xi$ and $\zeta$ are the Lorentz invariance violating terms for the nucleons and it is assumed that corresponding terms for the produced secondary pions are negligible. Note that in principle the values for $\xi=\xi_{ \pm}$and $\zeta=\xi_{ \pm}$can be different for the two nucleon helicities.

One can show that the same modified dispersion relation Eq. (5.1) leads to the same condition Eq. (5.4) for absorption of high energy gamma rays through $e^{+} e^{-}$pair production on the infrared, microwave or radio backgrounds, if one substitutes $p_{\mathrm{th}, 0}=m_{e}^{2} / \varepsilon, \alpha=\xi p_{\mathrm{th}, 0}^{3} /\left(8 m_{e}^{2} M_{\mathrm{Pl}}\right)$, $\beta=3 \zeta p_{\mathrm{th}, 0}^{4} /\left(16 m_{e}^{2} M_{\mathrm{Pl}}^{2}\right)$, where $m_{e}$ is the electron mass.

If $\xi_{ \pm}, \zeta_{ \pm} \simeq 1$ for at least one of the nucleon helicities, there is no real positive solution of Eq. (5.4) for the corresponding helicity, implying that the GZK process does not take place and consequently the GZK cutoff effect disappears completely [71]. Thus UHE nucleons and/or photons will be able to reach Earth from any distance. On the other hand, if recent indications for a suppression of the UHECR flux above $\simeq 4 \times 10^{19} \mathrm{eV}$ by the HiRes [19, 20] and Pierre Auger experiments [21, 22] are confirmed to be due to the GZK effect, as expected around these energies, then this will imply upper limits on the couplings $\xi_{ \pm}$and $\zeta_{ \pm}$for both nucleon helicities, thus probing specific Lorentz non-invariant theories. If $p_{\text {th }} \simeq p_{\text {th }, 0}$, one could conclude from Eq. (5.4) 
that $\alpha, \beta \lesssim 1$, which translates into $\left|\xi_{ \pm}\right| \lesssim 10^{-13}$ for the first order effects, and $\left|\zeta_{ \pm}\right| \lesssim 10^{-6}$ for the second order effects, when $\xi_{ \pm}=0$ [70,71]. These values correspond to values $|d| \lesssim 10^{-23}$ for the parameters of renormalizable VLI. Confirmation of a cut-off for $\mathrm{TeV}$ photons with next-generation $\gamma$-ray observatories would lead to somewhat weaker constraints [72].

More generally, modification of reaction kinematics or new reaction channels are expected whenever the terms on the right hand side of Eq. (5.1) become comparable to $m^{2}$, in rough numbers,

$$
\begin{aligned}
& d \gtrsim \frac{m^{2}}{2 E^{2}} \simeq 5 \times 10^{-23}\left(\frac{m}{\mathrm{GeV}}\right)^{2}\left(\frac{E}{10^{20} \mathrm{eV}}\right)^{-2}, \\
& \xi \gtrsim \frac{M_{\mathrm{P} 1} m^{2}}{E^{3}} \simeq 10^{-14}\left(\frac{m}{\mathrm{GeV}}\right)^{2}\left(\frac{E}{10^{20} \mathrm{eV}}\right)^{-3}, \\
& \zeta \gtrsim \frac{M_{\mathrm{Pl}}^{2} m^{2}}{E^{4}} \simeq 10^{-6}\left(\frac{m}{\mathrm{GeV}}\right)^{2}\left(\frac{E}{10^{20} \mathrm{eV}}\right)^{-4} .
\end{aligned}
$$

Note that by far the smallest parameter values would be probed by particles with the smallest mass, specifically the neutrino, $m \lesssim \mathrm{eV}$ at the highest energies. This makes the prospects of future detections of cosmogenic neutrinos, see, e.g., Fig. 1, very exciting also for VLI constraints.

In addition, the non-renormalizable terms in the dispersion relation Eq. (5.1) imply a change in the group velocity which for the first-order term leads to time delays over distances $r$ given by

$$
\Delta t \simeq \xi r \frac{E}{M_{\mathrm{Pl}}} \simeq \xi\left(\frac{r}{100 \mathrm{Mpc}}\right)\left(\frac{E}{\mathrm{TeV}}\right) \mathrm{sec} .
$$

For $|\xi| \sim 1$ such time delays could be measurable, for example, by fitting the arrival times of $\gamma$-rays arriving from $\gamma$-ray bursts to the predicted energy dependence.

We mention that if VLI is due to modification of the space-time structure expected in some theories of quantum gravity, for example, then the strict energy-momentum conservation assumed in the above discussion, which requires space-time translation invariance, is not guaranteed in general, and then the calculation of the modified particle interaction thresholds becomes highly non-trivial and non-obvious. Also, it is possible that a Lorentz non-invariant theory while giving a modified dispersion relation also imposes additional kinematic structures such as a modified law of addition of momenta. Indeed, Ref. [64] gives an example of a so-called $\kappa$-Minkowski non-commutative space-time in which the modified dispersion relation has the same form as in Eq. (5.1) but there is also a modified momentum addition rule which compensates for the effect of the modified dispersion relation on the particle interaction thresholds discussed above leaving the threshold momentum unaffected and consequently the GZK problem unsolved. In scenarios where the relativity of inertial frames is preserved by a non-linear representation of the Poincare group, thresholds are in general significantly modified only if the effective mass scale $M_{\mathrm{Pl}} / \xi$ is of the order of the unmodified threshold energy in the laboratory frame [73]. In another approach called "doubly special relativity" (DSR), the Lorentz transformations are modified in such a way that they leave invariant not only the speed of light, but also some minimal length scale identified with the Planck lenght [74].

There are several other fascinating effects of allowing a small VLI, some of which are relevant for the question of origin and propagation of UHECR. For example, any movement relative to the preferred frame defined by $u^{\mu}$ in Eq. (5.2) gives rise to spatial anisotropy. Clock comparison 
and spin precession experiments then lead to limits on the dimensionless parameters in Eq. (5.2) between $\mathscr{O}(1)$ and $\mathscr{O}\left(10^{-8}\right)$, depending on the particle [66].

Astrophysical observations can lead to comparable or even stronger limits: The observation of polarized $\mathrm{MeV}$ synchrotron radiation from electrons in the Crab nebula would imply the absence of vacuum Čerenkov radiation $e \rightarrow e \gamma$ for electrons up to energies $E \sim 1.5 \mathrm{PeV}[75,76]$. This process can become possible if the electron speed becomes larger than the speed of light at high energies and would lead to a limit $|\xi| \lesssim 2 \times 10^{-15}$ for the photon, significantly stronger than the before mentioned laboratory constraints $[75,76]$. However, the $\mathrm{MeV}$ photon polarization could not yet be confirmed [77] and the best existing upper limit is $|\xi| \lesssim 2 \times 10^{-7}$ [78], based on frequency dependent rotation of linear polarization (vacuum birefringence) of optical/UV photons of the afterglow from distant $\gamma$-ray bursts.

Recently we found that first and second order Lorentz invariance violating terms for photons of size $|\xi| \gtrsim 10^{-14}$ and $\zeta \gtrsim 10^{-6}$, respectively, in the convention of Eq. (5.1), would lead to a photon component in cosmic rays above $10^{19} \mathrm{eV}$ of order $20 \%$ [79]. This is in conflict with the latest experimental upper limits from the surface detector data of the Pierre Auger observatory which are $\sim 2 \%$ above $10^{19} \mathrm{eV}[80]$ which are consistent with predictions of standard astrophysical scenarios for unmodified pair production which are smaller than $\sim 1 \%$ around $10^{19} \mathrm{eV}$, and smaller than $\sim 10 \%$ around $10^{20} \mathrm{eV}[81,82]$. This suggests that LI breaking suppressed up to second order in the Planck scale are unlikely to phenomenologically viable for photons. LI breaking for photons is, therefore, also unlikely to play any role in $\mathrm{TeV} \gamma$-ray astrophysics: For example, recently the MAGIC telescope observed an intense flare from Markarian 501 in which photons in the $1.2-10$ $\mathrm{TeV}$ energy band arrived about 4 minutes after photons in the energy band $0.25-0.6 \mathrm{TeV}$. This was discussed as a potential signature of modified photon dispersion relations [83]. In the convention of Eq. (5.1), the required parameters are $\xi \sim 6$ or $\zeta \sim 1.6 \times 10^{15}$ for effects suppressed to first and second order in the Planck mass, respectively. This is in conflict with our constraints from $10^{19} \mathrm{eV}$ photons by a wide margin.

Constraints from highest energy cosmic and $\gamma$-rays basically rule out effects of order $E / M_{\mathrm{Pl}}$ which might be a challenge for certain quantum gravity scenarios [84]. Note that these current constraints on VLI parameters still allow strong modification of GZK kinematics by VLI parameters of the order given in Eq. (5.6).

\section{Acknowledgements}

This work has been supported by the European Science Foundation network programme "Quantum Geometry and Quantum Gravity".

\section{References}

[1] for a recent introduction to cosmic rays see, e.g., T. Stanev, High Energy Cosmic Rays (Springer 2004).

[2] F. Aharonian et al., Nature 432, 75 (2004).

[3] N. Sidro [MAGIC Collaboration], arXiv:astro-ph/0610945.

[4] J. R. Hoerandel, Astropart. Phys. 21, 241 (2004) [arXiv:astro-ph/0402356]. 
[5] R. Aloisio, V. Berezinsky, P. Blasi, A. Gazizov, S. Grigorieva and B. Hnatyk, Astropart. Phys. 27, 76 (2007) [arXiv:astro-ph/0608219].

[6] M. Lemoine, Phys. Rev. D 71, 083007 (2005) [arXiv:astro-ph/0411173].

[7] G. Sigl and E. Armengaud, JCAP 0510, 016 (2005) [arXiv:astro-ph/0507656].

[8] A. A. Watson, Nucl. Phys. Proc. Suppl. 151, 83 (2006) [arXiv:astro-ph/0410514].

[9] R. U. Abbasi et al. [The High Resolution Fly's Eye Collaboration], Astrophys. J. 622, 910 (2005) [arXiv:astro-ph/0407622].

[10] T. Abu-Zayyad et al. [HiRes-MIA Collaboration], Astrophys. J. 557, 686 (2001) [arXiv:astro-ph/0010652].

[11] D. Harari, S. Mollerach and E. Roulet, JCAP 0611, 012 (2006) [arXiv:astro-ph/0609294].

[12] for a recent review see, e.g., J. W. Cronin, Nucl. Phys. Proc. Suppl. 138, 465 (2005) [arXiv:astro-ph/0402487].

[13] W. S. Burgett and M. R. O’Malley, Phys. Rev. D 67, 092002 (2003) [arXiv:hep-ph/0301001].

[14] K. Shinozaki [AGASA Collaboration], Nucl. Phys. Proc. Suppl. 151, 3 (2006).

[15] C. B. Finley and S. Westerhoff, Astropart. Phys. 21, 359 (2004) [arXiv:astro-ph/0309159].

[16] C. B. Finley et al., Proc. 28th International Cosmic Ray Conference, Tsukuba, Japan, 1 (2003) 433.

[17] K. Greisen, Phys. Rev. Lett. 16, 748 (1966); G. T. Zatsepin and V. A. Kuzmin, JETP Lett. 4, 78 (1966) [Pisma Zh. Eksp. Teor. Fiz. 4, 114 (1966)].

[18] F. W. Stecker, Phys. Rev. Lett. 21, 1016 (1968).

[19] D. R. Bergman [HiRes Collaboration], Nucl. Phys. Proc. Suppl. 165, 19 (2007) [arXiv:astro-ph/0609453].

[20] R. Abbasi et al. [HiRes Collaboration], arXiv:astro-ph/0703099.

[21] T. Yamamoto [Pierre Auger Collaboration], arXiv:0707.2638 [astro-ph].

[22] M. Roth [Pierre Auger Collaboration], arXiv:0706.2096 [astro-ph].

[23] M. Takeda et al., Phys. Rev. Lett. 81, 1163 (1998) [arXiv:astro-ph/9807193]; N. Hayashida et al., arXiv:astro-ph/0008102; see also http ://www-akeno.icrr.u-tokyo.ac.jp/AGASA/.

[24] D. De Marco, P. Blasi and A. V. Olinto, Astropart. Phys. 20, 53 (2003) [arXiv:astro-ph/0301497].

[25] M. Teshima, Proceedings of CRIS 2006, Catania, Italy.

[26] R. U. Abbasi et al. [HiRes Collaboration], Astrophys. J. 636, 680 (2006) [arXiv:astro-ph/0507120].

[27] D. S. Gorbunov, P. G. Tinyakov, I. I. Tkachev and S. V. Troitsky, JETP Lett. 80, 145 (2004) [Pisma Zh. Eksp. Teor. Fiz. 80, 167 (2004)] [arXiv:astro-ph/0406654].

[28] The Pierre Auger Collaboration, Science 318, 939 (2007) [arXiv:0711.2256 [astro-ph]].

[29] T. E. Clarke, P. P. Kronberg and H. Boehringer, Astrophys. J. 547, L111 (2001) [arXiv:astro-ph/0011281].

[30] M. Johnston-Hollitt and R. D. Ekers, arXiv:astro-ph/0411045.

[31] F. Govoni, M. Murgia, L. Feretti, G. Giovannini, K. Dolag and G. B. Taylor, arXiv:astro-ph/0608433.

[32] G. Sigl, F. Miniati and T. A. Ensslin, Phys. Rev. D 70, 043007 (2004) [arXiv:astro-ph/0401084]. 
[33] G. Sigl, F. Miniati and T. Ensslin, Nucl. Phys. Proc. Suppl. 136, 224 (2004) [arXiv:astro-ph/0409098].

[34] K. Dolag, D. Grasso, V. Springel and I. Tkachev, JCAP 0501, 009 (2005) [arXiv:astro-ph/0410419].

[35] A. Elyiv, arXiv:astro-ph/0611696.

[36] G. Sigl, JCAP 0408, 012 (2004) [arXiv:astro-ph/0405549]; E. Armengaud, G. Sigl and F. Miniati, Phys. Rev. D 72, 043009 (2005) [arXiv:astro-ph/0412525].

[37] for recent reviews see, e.g., F. Halzen and D. Hooper, Rept. Prog. Phys. 65, 1025 (2002) [arXiv:astro-ph/0204527]; A. B. McDonald, C. Spiering, S. Schonert, E. T. Kearns and T. Kajita, Rev. Sci. Instrum. 75, 293 (2004) [arXiv:astro-ph/0311343].

[38] for a recent short review see, e.g., H. J. Völk, arXiv:astro-ph/0603501.

[39] M. D. Kistler and J. F. Beacom, Phys. Rev. D 74, 063007 (2006) [arXiv:astro-ph/0607082].

[40] P. Bhattacharjee and G. Sigl, Phys. Rept. 327, 109 (2000) [arXiv:astro-ph/9811011].

[41] see http://apcauger.in2p3.fr//CRPropa.

[42] E. Armengaud, G. Sigl, T. Beau and F. Miniati, to appear in Astropart. Phys. (2007) [arXiv:astro-ph/0603675].

[43] D. V. Semikoz and G. Sigl, JCAP 0404, 003 (2004) [arXiv:hep-ph/0309328].

[44] E. Waxman and J. N. Bahcall, Phys. Rev. D 59, 023002 (1999) [arXiv:hep-ph/9807282]; J. N. Bahcall and E. Waxman, Phys. Rev. D 64, 023002 (2001) [arXiv:hep-ph/9902383].

[45] J. P. Rachen, R. J. Protheroe and K. Mannheim, arXiv:astro-ph/9908031.

[46] J. Candia and E. Roulet, JCAP 0309, 005 (2003) [arXiv:astro-ph/0306632].

[47] A. W. Strong, I. V. Moskalenko and O. Reimer, arXiv:astro-ph/0306345.

[48] see, e.g., T. Stanev, arXiv:astro-ph/0607515.

[49] S. Ando and J. F. Beacom, Phys. Rev. Lett. 95, 061103 (2005) [arXiv:astro-ph/0502521].

[50] T. Kashti and E. Waxman, Phys. Rev. Lett. 95, 181101 (2005) [arXiv:astro-ph/0507599].

[51] P. D. Serpico, Phys. Rev. D 73, 047301 (2006) [arXiv:hep-ph/0511313].

[52] D. Hooper, Czech. J. Phys. 56, A337 (2006) [arXiv:hep-ph/0510097].

[53] Z. Fodor, S. D. Katz, A. Ringwald and H. Tu, Phys. Lett. B 561, 191 (2003) [arXiv:hep-ph/0303080].

[54] G. Domokos and S. Kovesi-Domokos, Phys. Rev. Lett. 82, 1366 (1999) [arXiv:hep-ph/9812260].

[55] N. Arkani-Hamed, S. Dimopoulos and G. R. Dvali, Phys. Lett. B 429, 263 (1998) [arXiv:hep-ph/9803315]; I. Antoniadis, N. Arkani-Hamed, S. Dimopoulos and G. R. Dvali, Phys. Lett. B 436, 257 (1998) [arXiv:hep-ph/9804398]; N. Arkani-Hamed, S. Dimopoulos and G. R. Dvali, Phys. Rev. D 59, 086004 (1999) [arXiv:hep-ph/9807344].

[56] J. L. Feng and A. D. Shapere, Phys. Rev. Lett. 88, 021303 (2002) [arXiv:hep-ph/0109106].

[57] M. Kachelriess and M. Plumacher, Phys. Rev. D 62, 103006 (2000) [arXiv:astro-ph/0005309].

[58] D. A. Morris and A. Ringwald, Astropart. Phys. 2, 43 (1994) [arXiv:hep-ph/9308269].

[59] C. Tyler, A. V. Olinto and G. Sigl, Phys. Rev. D 63, 055001 (2001) [arXiv:hep-ph/0002257]. 
[60] see, e.g., L. A. Anchordoqui, J. L. Feng, H. Goldberg and A. D. Shapere, Phys. Rev. D 65, 124027 (2002) [arXiv:hep-ph/0112247]; L. A. Anchordoqui, J. L. Feng, H. Goldberg and A. D. Shapere, Phys. Rev. D 66, 103002 (2002) [arXiv:hep-ph/0207139].

[61] A. Kusenko and T. J. Weiler, Phys. Rev. Lett. 88, 161101 (2002) [arXiv:hep-ph/0106071].

[62] H. Sato and T. Tati, Prog. Theor. Phys. 47, 1788 (1972); D. A. Kirzhnits and V. A. Chechin, Sov. J. Nucl. Phys. 15, 585 (1972); L. Gonzalez-Mestres, arXiv:hep-th/0208064.

[63] S. R. Coleman and S. L. Glashow, Phys. Lett. B 405, 249 (1997) [arXiv:hep-ph/9703240];

S. R. Coleman and S. L. Glashow, Phys. Rev. D 59, 116008 (1999) [arXiv:hep-ph/9812418].

[64] G. Amelino-Camelia and T. Piran, Phys. Lett. B 497, 265 (2001) [arXiv:hep-ph/0006210].

[65] D. Colladay and V. A. Kostelecky, Phys. Rev. D 58, 116002 (1998) [arXiv:hep-ph/9809521].

[66] R. C. Myers and M. Pospelov, Phys. Rev. Lett. 90, 211601 (2003) [arXiv:hep-ph/0301124].

[67] see, e.g., S. Bernadotte and F. R. Klinkhamer, Phys. Rev. D 75, 024028 (2007) [arXiv:hep-ph/0610216].

[68] see, e.g., J. R. Ellis, N. E. Mavromatos and D. V. Nanopoulos, Phys. Rev. D 62, 084019 (2000) [arXiv:gr-qc/0006004].

[69] S. Groot Nibbelink and M. Pospelov, Phys. Rev. Lett. 94, 081601 (2005) [arXiv:hep-ph/0404271].

[70] R. Aloisio, P. Blasi, P. L. Ghia and A. F. Grillo, Phys. Rev. D 62, 053010 (2000) [arXiv:astro-ph/0001258].

[71] F. W. Stecker and S. T. Scully, Astropart. Phys. 23, 203 (2005) [arXiv:astro-ph/0412495].

[72] see, e.g., R. J. Protheroe and H. Meyer, Phys. Lett. B 493, 1 (2000) [arXiv:astro-ph/0005349]; T. Jacobson, S. Liberati and D. Mattingly, Phys. Rev. D 66, 081302 (2002) [arXiv:hep-ph/0112207].

[73] J. Magueijo and L. Smolin, Phys. Rev. D 67, 044017 (2003) [arXiv:gr-qc/0207085].

[74] G. Amelino-Camelia, Phys. Lett. B 510, 255 (2001) [arXiv:hep-th/0012238].

[75] T. A. Jacobson, S. Liberati, D. Mattingly and F. W. Stecker, Phys. Rev. Lett. 93, 021101 (2004) [arXiv:astro-ph/0309681].

[76] T. Jacobson, S. Liberati and D. Mattingly, Lect. Notes Phys. 669, 101 (2005) [arXiv:hep-ph/0407370].

[77] S. Liberati, PoS P2GC, 018 (2007) [arXiv:0706.0142 [gr-qc]].

[78] Y. Z. Fan, D. M. Wei and D. Xu, Mon. Not. Roy. Astron. Soc. 376, 1857 (2006) [arXiv:astro-ph/0702006].

[79] M. Galaverni and G. Sigl, arXiv:0708.1737 [astro-ph], to appear in Phys. Rev. Lett.

[80] M. D. Healy for the Pierre Auger Collaboration, arXiv:0710.0025 [astro-ph].

[81] G. Sigl, Phys. Rev. D 75, 103001 (2007) [arXiv:astro-ph/0703403].

[82] G. B. Gelmini, O. Kalashev and D. V. Semikoz, arXiv:0706.2181 [astro-ph].

[83] J. Albert et al. [MAGIC Collaboration], arXiv:0708.2889 [astro-ph].

[84] for a non-technical overview see M. Pospelov and M. Romalis, Physics Today July 2004, p. 40. 